\title{
Xueshuantong injection (lyophilized) combined with salvianolate lyophilized injection protects against focal cerebral ischemia/reperfusion injury in rats through attenuation of oxidative stress
}

\author{
Fu-jiang WANG ${ }^{1,2}$, Shao-xia WANG ${ }^{1}$, Li-juan $\mathrm{CHAl}^{2}$, Yue ZHANG ${ }^{2}$, Hong GUO ${ }^{2}$, Li-min $\mathrm{HU}^{2, *}$ \\ ${ }^{1}$ Tianjin State Key Laboratory of Modern Chinese Medicine, Tianjin University of Traditional Chinese Medicine, Tianjin 300193, China; \\ ${ }^{2}$ Key Laboratory of Formula of Traditional Chinese Medicine (Tianjin University of Traditional Chinese Medicine), Ministry of Education, \\ Tianjin 300193, China
}

\begin{abstract}
Salvianolate lyophilized injection (SLI) and Xueshuantong injection (lyophilized) (XST) are two herbal standardized preparations that have been widely used in China for the treatment of acute cerebral infarction. In this study, we investigated the neuroprotective effects of SLI combined with XST in a rat model of middle cerebral artery occlusion-reperfusion (MCAO/R). Wistar rats were subjected to $1.5 \mathrm{~h}$ of MCAO followed by reperfusion for $3 \mathrm{~h}$, then were treated with SLI or XST alone, or with their combinations via tail vein injection daily for $3 \mathrm{~d}$. Edaravone (EDI, $6 \mathrm{mg} \cdot \mathrm{kg}^{-1} \cdot \mathrm{d}^{-1}$ ) was used as a positive control drug, We showed that administration of a combination of 1X1S (XST $100 \mathrm{mg} \cdot \mathrm{kg}^{-1} \cdot \mathrm{d}^{-1}$ plus SLI $21 \mathrm{mg} \cdot \mathrm{kg}^{-1} \cdot \mathrm{d}^{-1}$ ) more effectively protected the ischemic brains than SLI or XST used alone. Administration of 1X1S not only significantly decreased neurological deficit scores and infarct volumes and increased regional cerebral blood flow, but also inhibited the activation of both microglia and astrocytes in the hippocampus. Furthermore, administration of 1X1S significantly decreased the levels of MDA and ROS with concomitant increases in the levels of antioxidant activity (SOD, CAT and GSH) in the brain tissues as compared with SLI and XST used alone. Moreover, administration of 1X1S remarkably upregulated the expression of Nrf-2, HO-1 and NQO-1, and downregulated the expression of Keap1 and facilitated the nuclear translocation of Nrf-2 in the brain tissues as compared with XST used alone. Our study demonstrates that a combination of 1X1S effectively protects MCAO/R injury via suppressing oxidative stress and the Nrf-2/Keap1 pathway.
\end{abstract}

Keywords: stroke; cerebral ischemia-reperfusion injury; oxidative stress; Nrf-2/Keap1 pathway; Salvianolate lyophilized injection; Xueshuantong injection (lyophilized); edaravone

Acta Pharmacologica Sinica (2018) 39: 998-1011; doi: 10.1038/aps.2017.128; published online 12 Oct 2017

\section{Introduction}

Stroke still remains a major cause of death and disability worldwide with serious long-term physical and cognitive disabilities that contribute to the rising costs of health care, despite all efforts of neuroscience community ${ }^{[1,2]}$. Nearly $60 \%-$ $70 \%$ of stroke is triggered by ischemia ${ }^{[3]}$, which is characterized by the occlusion of blood vessels through the formation of an obstructive thrombus or embolus. Currently, advanced intravascular procedures and thrombolytic treatment with tissue plasminogen activator agent are the only existing phar-

\footnotetext{
${ }^{*}$ To whom correspondence should be addressed. E-mail hulimin@tjutcm.edu.cn Received 2017-02-25 Accepted 2017-08-08
}

macological therapies for the acute phase of stroke, which can effectively reduce functional deficits if given within an optimal time window in stroke patients ${ }^{[4]}$. However, the challenge of reperfusion injury remains for this thrombolytic treatment. Reperfusion after ischemia leads to neuronal injury and death, including the release of nitric oxide, excitatory amino acids, cytokines, and free radicals, mitochondrial respiratory enzymes damage, microglia activation, cerebral microcirculatory disturbance and even death of neurons ${ }^{[5,6]}$. Therefore, protecting the brain from reperfusion injury after ischemia is an alternative for therapy of stroke.

Both the occlusion of blood supply to the brain and cerebral ischemia result in the rapid onset of neurological injury. The treatment of ischemic stroke has important strategic 
approaches, including the repair of infarct damage and the improvement of functional recovery. Numerous studies have confirmed that the decrease of the regional cerebral blood flow (rCBF) and a series of oxidative stress and inflammatory reactions are major contributors to the pathogenesis of ischemic stroke. Oxidative stress, during which excessive reactive oxygen species (ROS) are produced, is a core pathological component of brain ischemia-reperfusion injury causing neuronal malfunction and cell death ${ }^{[7,8]}$. Nuclear factor erythroid 2-related factor 2 (Nrf-2), a newly discovered gene transcription factor with high sensitivity to oxidative stress, is a major component in the kelch-like ECH-associated protein 1 (Keap1)-Nrf-2/antioxidant response element (ARE) antioxidant system. In physiological conditions, Nrf-2 is kept inactivated in association with Keap1 in the cytoplasm. When oxidative stress occurs, ROS may bring conformational changes to cause Nrf-2 activation. Nrf-2 dissociates from Keap1 and combines with ARE in the nucleus to initiate gene expression of heme-oxygenase-1 (HO-1) and other antioxidant enzymes, such as $\mathrm{NAD}(\mathrm{P}) \mathrm{H}$ quinone oxidoreductase (NQO-1), superoxide dismutase (SOD), catalase (CAT), malondialdehyde (MDA) and glutathione (GSH) ${ }^{[9,10]}$. The Nrf-2/Keap1 pathway has been shown to play an important neuroprotective role in brain injury after ischemic stroke $\mathrm{e}^{[11,12]}$.

In contrast with Western countries, China has many herbs and herbal standardized preparations for the clinical treatment of ischemic stroke. Danshen, the dried root or rhizome of Salviae miltiorrhizae Bge (SM), and Sanqi, the root of Panax notoginseng (Burk), have been widely used in China or other oriental countries for the treatment of cerebrovascular and cardiovascular conditions, such as to cure heart disease, ischemic stroke, and acute intracerebral hemorrhage $\mathrm{e}^{[13,14]}$. Salvianolate lyophilized injection (SLI), which is composed of the Danshen aqueous extraction, has been approved in the treatment of stroke by the State Food and Drug Administration in China since 2011. Furthermore, the salutary effect of the SLI components on cerebrovascular disease is widely reported ${ }^{[15,16]}$. Xueshuantong for injection (lyophilized) (XST) is a standardized product extracted from Sanqi. SLI is used extensively for the treatment of cardiovascular disease, cerebrovascular disease, and diabetes in China ${ }^{[17]}$.

Many studies suggest that SLI has increased myocardial microvascular reflow, promoted cardiac functional recovery and alleviated hepatocyte injury through attenuation of oxidative stress, and XST had decreased proteinuria through its suppression of oxidative stress ${ }^{[18-20]}$. Although those studies reported that the SLI and XST used alone have an oxidative stress effect, there is no report on the effect of SLI and XST protecting against ischemic injury in $\mathrm{MCAO} / \mathrm{R}$ rats through its suppression of oxidative stress. Therefore, we wanted to study the effects of SLI and XST in protecting against antioxidative stress in $\mathrm{MCAO} / \mathrm{R}$ rats. Additionally, we investigated whether the combined use strategy can compensate for their own disadvantages in the treatment of stroke or have stronger effects than the drug used alone.

\section{Materials and methods Drugs and reagents}

SLI, provided by Tianjin Tasly Pharmaceutical Co, Ltd (Tianjin, China), was authenticated and standardized in accordance with the Pharmacopoeia of China 2011. SLI were multiple salvianolic acids, primarily including salvianolic acid B (Sal B) $(62.1 \%)$, salvianolic acid E (Sal E) $(1.8 \%)$, lithospermic acid (LA) $(3.7 \%)$ and rosmarinic acid (RA) $(4.5 \%)^{[21-23]}$. XST was obtained from Wuzhou Pharmaceutical Co, Ltd (Wuzhou, China). The manufacturing technology was taken from the "Pharmacopoeia of China 2010." The HPLC fingerprint shows that it contains ginsenosides Rg1 (48.1\%), ginsenosides Rb1 (27.8\%), notoginsenoside R1 (11.1\%), ginsenosides Re (5.5\%), ginsenosides $\mathrm{Rd}(1.3 \%)$, notoginsenoside $\mathrm{Ra}(1.1 \%)$, and 20 -Oglucoginsenoside $\operatorname{Rf}(0.7 \%)^{[24,25]}$. The structures of these constituents are shown in Figure 1. In this study, SLI and XST were freshly prepared in $0.9 \%$ normal saline before use. Edaravone injection (batch number: H20031342) was purchased from Nanjing Simcere Pharma Co, Ltd (Nanjing, China).

Chloral hydrate (batch number: Q/12HB 4218-2009) was purchased from Tianjin Kermel Chemical Reagent Co, Ltd (Tianjin, China), freshly prepared to a $3.5 \%$ solution with saline before experiments. Nissl stain kit, H\&E kit, DAB substrate kit and crystal violet stain kit were purchased from Boster (Wuhan, China). BCA protein assay kit was purchased from CWBIO (China). Rabbit anti-rat monoclonal antibodies for glial fibrillary acidic protein (GFAP, ab7260), ionized calcium binding adaptor molecule-1 (IBA-1, ab178680), Nrf-2 (ab31163), NQO-1 (ab34173), HO-1 (ab68477), Keap1 (ab66620) and $\beta$-actin (ab8227) were purchased from Abcam (Massachusetts, USA). Secondary antibodies were purchased from Zhongshan Golden Bridge Biotechnology Co, Ltd (Beijing, China). Hoechst 33258 was bought from Beyotime (Haimen, China). TRIzol reagent was purchased from Life Technologies (USA).

\section{Animals}

Adult male Wistar rats (10 to 13 weeks old, 250-300 g) were purchased from Vital River Laboratory Animal Technology Co, Ltd (Beijing, China). The animals were housed in an airconditioned room (temperature, $22-25{ }^{\circ} \mathrm{C}$; relative humidity, $55 \% \pm 5 \%$ ), and kept on a light/dark cycle of 12/12 h. Free access to food and drinking water was allowed throughout the study. Each rat was used only once. All animal manipulations were performed in accordance with European Community Guidelines for Animal Care and approved by the Committee at Tianjin University of Traditional Chinese Medicine in China (TCM-LAEC2015028). All experiments involving animals are reported in accordance with the animal research: reporting in vivo experiments (ARRIVE) guidelines.

\section{Focal cerebral ischemia-reperfusion injury model}

Focal cerebral ischemia-reperfusion was induced with minor modification of intraluminal MCAO as previously described $^{[26-28]}$. The left common carotid artery (CCA) was exposed at its bifurcation using a midline cervical incision. The external carotid artery (ECA), internal carotid artery 


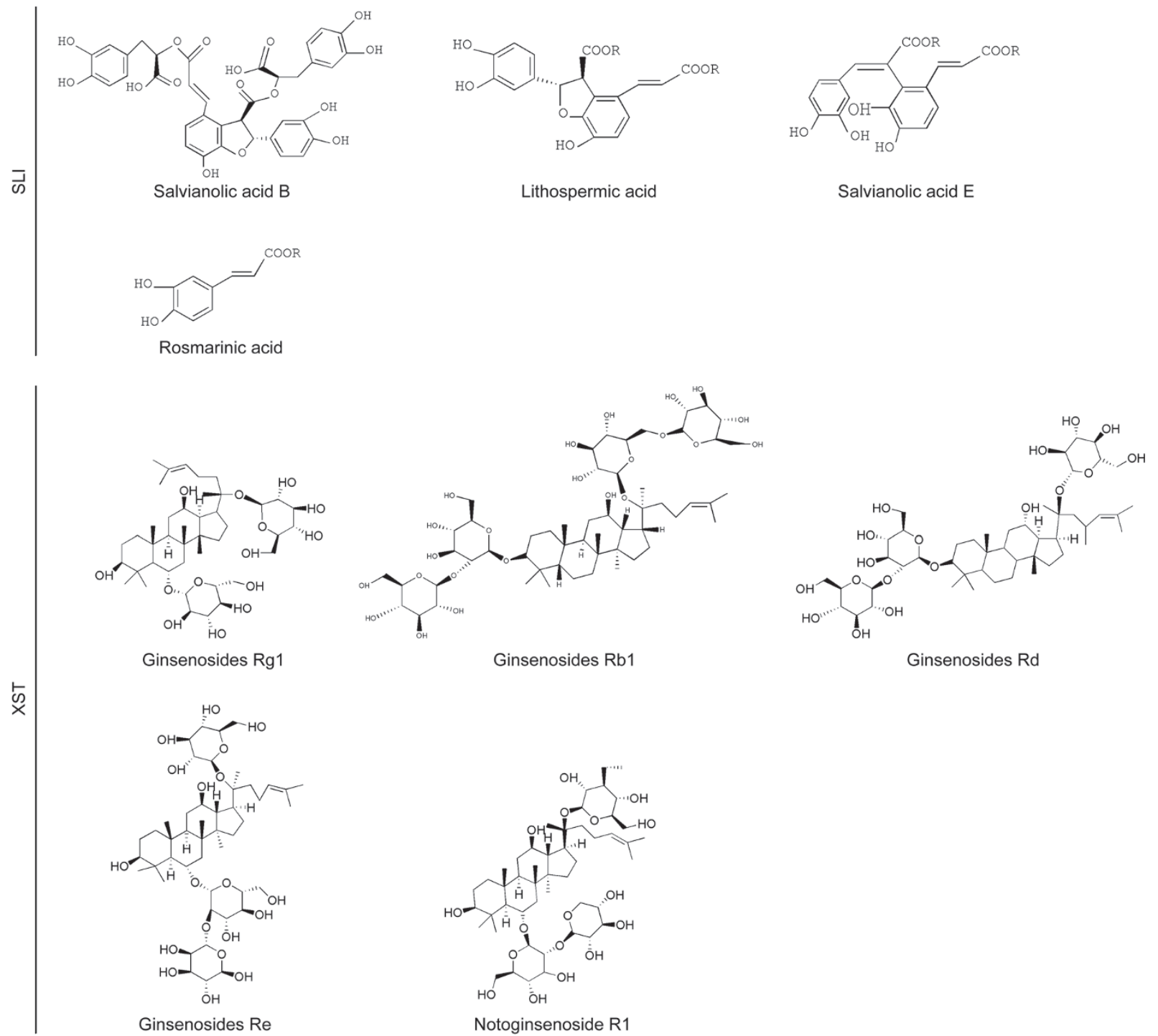

Figure 1. Chemical structures of main constituents in SLI and XST.

(ICA), and common carotid artery (CCA) were ligated using a 4-0 silk suture. The ECA was later transected, and a 3-0 nylon monofilament suture was inserted from the ECA into the ICA until reaching the origin of the MCA. The suture was advanced into the ICA until the MCA flow was $<30 \%$ of baseline in order to exclude incomplete ischemia. The focal cerebral ischemia was confirmed by obvious changes of $\mathrm{rCBF}$ on a laser Doppler flow meter $(2 \mathrm{~mm}$ posterior and $6 \mathrm{~mm}$ lateral to bregma on the right parietal skull). After $1.5 \mathrm{~h}$ of MCA occlusion, reperfusion was simulated by gently removing the filament. Finally, the distal two collar sutures were further tightened and trimmed. The animals were allowed food and water ad libitum. Core temperature was recorded continuously using a rectal probe, and a heating pad was used to maintain rectal temperature at $36.5-37.5^{\circ} \mathrm{C}$.

\section{Drug administration}

The rats were randomly divided into the following 7 groups: control group (NOR), MCAO/R group (MOD), SLI group $(21 \mathrm{mg} / \mathrm{kg}$, SLI), XST group (100 mg/kg, XST), SLI combined with XST group (XST $100 \mathrm{mg} / \mathrm{kg}$ : SLI $21 \mathrm{mg} / \mathrm{kg}$, 1X1S), SLI combined with XST group (XST $50 \mathrm{mg} / \mathrm{kg}$ : SLI $10.5 \mathrm{mg} / \mathrm{kg}$, $1 / 2 \times 1 / 2 S$ ), and edaravone group (as a positive control drug, $6 \mathrm{~mL} / \mathrm{kg}$, EDI). SLI and XST were freshly dissolved in normal saline before use. SLI and XST initially used intravenous injection (iv) $3 \mathrm{~h}$ after $\mathrm{MCAO} / \mathrm{R}$ and daily for $3 \mathrm{~d}$. The shamoperated group and model-vehicle group were treated with isodose saline. In all experiments, the data were collected by a blinded, randomized and controlled design. Number of animals in each group for determination of each parameter can be observed in Table 1.

Measurement of weight, neurological deficit score and mortality Body weight was monitored before surgery ( $0 \mathrm{~d})$ and at $\mathrm{d} 3$ (3 d) after surgery using a CS2000 Compact Digital Scale (Ohaus, Parsippany, NJ, USA). A modified neurological severity score 
Table 1. Number of animals for different experimental groups and various parameters at $3 \mathrm{~d}$ after administration.

\begin{tabular}{|c|c|c|c|c|c|c|c|c|}
\hline & NOR & MOD & EDI & SLI & XST & $1 \times 1 S$ & $1 / 2 \times 1 / 2 S$ & Total \\
\hline H\&E, Nissl stain and crystal violet & 12 & 12 & 12 & 12 & 12 & 12 & 12 & 84 \\
\hline Cerebral blood flow & 16 & 16 & 16 & 16 & 16 & 16 & 16 & 112 \\
\hline Measurement SOD, MDA, CAT, GSH and ROS & 5 & 5 & 5 & 5 & 5 & 5 & 0 & 30 \\
\hline Immunohistochemistry and Immunofluorescent & 4 & 4 & 4 & 4 & 4 & 4 & 4 & 28 \\
\hline Western blot and PCR analysis & 4 & 4 & 4 & 4 & 4 & 4 & 0 & 24 \\
\hline
\end{tabular}

(mNSS) evaluation was performed before $\mathrm{MCAO} / \mathrm{R}$ and at $3 \mathrm{~d}$ after MCAO by an investigator who was blinded to the experimental groups ${ }^{[29]}$. mNSS is a composite of motor, sensory, balance and reflex tests. The detailed methods and scorings for these components are listed in Table 2. Neurological function was graded on a scale of 0 to 18 (normal score 0 ; maximal deficit score 18) with one point awarded for the exhibition of specific abnormal behavior or for lack of a tested reflex. A greater impairment of normal function results in a higher score. The number of dead animals in each group was counted, and the mortality rate is presented as a percentage of rats that died $3 \mathrm{~d}$ after $\mathrm{MCAO} / \mathrm{R}$ to the total animals in each group.

\section{Regional cerebral blood flow (rCBF) measurements}

After $3 \mathrm{~d}$, the rats were deeply anesthetized with an intraperitoneal injection of chloral hydrate, and core body temperature was maintained at $37^{\circ} \mathrm{C}$ to $38^{\circ} \mathrm{C}$ by a heating pad; animals underwent a right unilateral craniotomy. Regional CBF within the right hemisphere was measured using laser Doppler flowmetry. The data were analyzed using a moorLDIMeasV60 data acquisition and analysis system.

\section{Measurement of SOD, CAT, MDA and GSH}

SOD, CAT, MDA and GSH in brain tissues were determined with kits following the manufacturer's instructions (Nanjing Jiancheng Bioengineering Institute, China).

\section{Detection of ROS generation}

ROS detection was performed as previously described ${ }^{[30]}$. Briefly, the brain homogenates were diluted with ice-cold phosphate-buffered saline (PBS) at a 1:20 ratio and after adding 2',7'-dichlorofluorescein diacetate (DCFH-DA, $10 \mu \mathrm{mol} / \mathrm{L}$ ), this mixture was incubated at $37{ }^{\circ} \mathrm{C}$ for $45 \mathrm{~min}$ for the conversion of DCFH-DA to 2',7'-dichlorofluorescein (DCF). Excitation was measured at $485 \mathrm{~nm}$, and emission was measured at $525 \mathrm{~nm}$. ROS formation was quantified from a DCF standard curve, and data were expressed as pmole DCF formed/min/mg protein.

\section{Nissl and H\&E staining}

After $3 \mathrm{~d}$, the rats were deeply anesthetized and killed by cardiac perfusion with $250 \mathrm{~mL}$ normal saline and $400 \mathrm{~mL}$ $4 \%$ paraformaldehyde successively. Next, the brains were
Table 2. Components of the modified neurological severity score (mNSS).

Methods and observations for specific deficits

Scores

Motor tests

Raising rat by tail

Normal response-balanced suspension 0

Flexion of forelimb

Hindlimb flexion

Head moves 10 degrees off vertical axis within $30 \mathrm{~s}$

Place rat on floor

Normal walk

Inability to walk straight

Circling toward paretic side

Falls down to paretic, ipsilateral side

Sensory tests

Placing Test

Normal response-forelimb placed on table

No forelimb placement in response to contralateral

whisker stimulation

Proprioceptive test

Normal response-Forepaw resistance to contralateral lateral push

No forelimb resistance to contralateral lateral push

tactile stimulation

Balance tests

Balances with steady posture 0

Grasps side of the beam 1

Hugs beam and 1 limb falls down from beam

Hugs beam and 2 limbs fall down from beam, or spins

Attempts to balance on beam but falls off ( $>40 \mathrm{~s}$ )

Attempts to balance on beam but falls off ( $>20 \mathrm{~s}$ )

Falls off; no attempt to balance or hang on to beam ( $<20 \mathrm{~s})$

Reflex absence and abnormal movements

Cotton Swab into ear canal

Normal response-head shake/pinna reflex 0

Lack of pinna reflex

Cotton Swab touches cornea

Normal response-eye closes/cornea reflex 0

Lack of corneal reflex

Loud hand clap

Normal response-Jumping movement/startle reflex 0

Lack of startle reflex

Observe daily for $5 \mathrm{~min}$

Normal response-no unusual motor behavior 0

Seizures, myoclonus, and/or myodystony
1

1

1

3

0

1

2

3

0 
rapidly removed and post-fixed in the same fixative for $7 \mathrm{~d}$ at $4{ }^{\circ} \mathrm{C}$. The brains were dehydrated in graded ethanol and xylene followed by being embedded in paraffin and sectioned into slices of $10 \mu \mathrm{m}$ on a rotary microtome. To evaluate brain injury by Nissl staining, slices were dewaxed, dehydrated, and stained with $1 \%$ toluidine blue at $50^{\circ} \mathrm{C}$ for $1 \mathrm{~h}$. In the present study, five random fields in the penumbra of ischemic cortex were captured in each section with a light microscope. The number of stained cells per field were calculated under higher magnification (100x) by an investigator blinded to experimental design. The positively stained cells were counted with Image-Pro Plus 6.0 (Media Cybernetics, Bethesda, MD, USA). Brain histology was measured using H\&E staining under light microscopy (Leica Microsystems) in all animals $3 \mathrm{~d}$ after stroke.

\section{Measurement of lesion volumes}

The brains were fixed by cardiac perfusion with saline followed by perfusion and immersion in $4 \%$ paraformaldehyde before being embedded in paraffin. Five coronal sections of tissue were processed and stained with crystal violet for calculation of volumes of cerebral infarction and presented as a percentage of the lesion compared with the contralateral hemisphere. The sections were examined under a light microscope (Leica Microsystems). Lesion volume was analyzed with ImageJ software (Wayne Rasband, National Institutes of Health, USA). Lesion percent was calculated with the formula lesion percent $(\%)=($ ipsilateral ischemic hemisphere volumecontralateral ischemic hemisphere volume)/contralateral ischemic hemisphere volume $\times 100$.

\section{Immunohistochemistry analysis}

Brain sections were immersed in $0.3 \%$ hydrogen peroxide to block intrinsic peroxidase and were treated with $5 \%$ bovine serum to block any nonspecific antibody responses; the sections were incubated with primary antibodies against IBA-1 (1:200) and GFAP (1:200), then incubated with secondary antibody (1:200) for $1 \mathrm{~h}$ at $37^{\circ} \mathrm{C}$. Immunostaining was visualized by using a DAB substrate kit. The numbers of IBA- 1 and GFAP per section were counted by an investigator who was blinded to the experimental groups. Images were captured through at least five randomly selected fields from three separate sections of each sample using a light microscope (Leica DM750M, Wetzlar, Germany) at magnification of 200×. The mean value of five fields for IBA- 1 and GFAP were calculated for the statistical analysis.

\section{Protein extraction and Western blotting}

Rats survived for $3 \mathrm{~d}$ after stroke onset was killed by overdose of isoflurane. Samples were obtained from the ischemic penumbra. Protein was extracted from brain tissues, and the protein concentration of each sample was determined using the BCA Protein Assay Kit. Next, $10 \mu \mathrm{g}$ of the samples were loaded per lane. The primary antibody against GFAP (1:1000), IBA-1 (1:1000), Nrf-2 (1:1000), HO-1 (1:1000), NQO-1 (1:1000), Keap1 (1:1000) and $\beta$-actin (1:1000) were used. $\beta$-Actin was used as a control. After washing with TBS-T, the membrane was incubated with horseradish peroxidase-conjugated secondary antibody (1:10 000) at room temperature for $60 \mathrm{~min}$. The relative intensities of the bands were measured by ImageJ software.

\section{RNA isolation and quantitative real-time qPCR}

Seventy-two hours after MCAO, rats were deeply anesthetized and perfused through the heart with cold PBS. Total RNA was isolated using TRIzol reagent and processed for cDNA followed by quantitative real-time polymerase chain reaction (PCR) as previously described ${ }^{[31]}$. The specific primer pairs (Sangon Technology Co, Ltd, Shanghai, China) are listed in Table 3. The mRNA levels of each cytokine were normalized to the level of $\beta$-actin, and the results were expressed as fold change of the threshold cycle $(\mathrm{Ct})$ value relative to shamoperated controls using the $2^{-\Delta \Delta \mathrm{Ct}}$ method. All samples were analyzed in triplicate.

\section{Immunofluorescence analysis}

Immunofluorescence staining for Nrf-2 and Keap1 was performed. Polyclonal antibodies against Nrf-2 and Keap1 were diluted to 1:200 and 1:100, respectively, in phosphate-buffered saline. Each section was washed and incubated with secondary antibody Alexa Flur488 mouse anti-rabbit IgG (1:200) and rhodamine (TRITC) goat anti-rabbit IgG (1:200), respectively, at $37^{\circ} \mathrm{C}$ for $1 \mathrm{~h}$. Hoechst $33258(50 \mu \mathrm{L})$ was applied to stain the nucleus. Images were captured using a confocal microscope (Nikon, Japan). Five non-overlapping fields of one slice were randomly observed under a magnification of $10 \times 10$ or $10 \times 20$ in images. To quantitatively analyze protein expression, ImagePro Plus software was applied.

\section{Statistical analysis}

The results are expressed as the mean \pm standard deviation (SD). Differences between groups were evaluated by one-way

Table 3. The specific primer pairs used in polymerase chain reaction.

\begin{tabular}{cl}
\hline Gene & Primer pair (5'-3') \\
& F, forward; R, reverse \\
\hline \multirow{2}{*}{ 3-Actin } & F 5'-GTAAAGACCTCTATGCCAACA-3' \\
& R 5'-GGACTCATCGTACTCCTGCT-3' \\
GFAP & F 5'-TTGTTTGCTAGGCCCAATTC-3' \\
& R 5'-CCTCGGGATCTTTTCCTTTC-3' \\
IBA-1 & F 5'-TCGTCATCTCCCCACCTAAG-3' \\
& R 5'-TCCATTGCCATTCAGATCAA-3' \\
Nrf-2 & F 5'-CTGCTGCCATTAGTCAGTCG-3' \\
& R 5'-GCCTTCAGTGTGCTTCTGGT-3' \\
HO-1 & F 5'-CAGAGTTTCTTCGCCAGAGG-3' \\
& R 5'-TGAGTGTGAGGACCCATCG-3' \\
NQ0-1 & F 5'-TCCAGAAACGACATCACAGG-3' \\
& R 5'-AGCTACAATATCCGGGCTCA-3' \\
Keap1 & F 5'-CACCAGGGCAGGATCTAC-3' \\
& R 5'-TTGCTTCCGACAGGGTTC-3'
\end{tabular}


analysis of variance (ANOVA) with LSD post hoc analysis. SPSS software version 17.0 was used for statistical analysis; $P$-values $<0.05$ were considered to be statistically significant and extremely significant when $P<0.01$.

\section{Results}

1X1S increases body weight and improves behavioral outcomes in $\mathrm{l} / \mathrm{R}$-treated rats

After $3 \mathrm{~d}$ of treatment with SLI, XST, EDI, 1X1S and saline, we evaluated the effects of SLI, XST, EDI and 1X1S in MCAO/R rats on body weight, neurological deficits and survival (Figure 2). As shown in Figure 2B-2D, the MOD group exhibited significantly decreased body weight, which was significantly attenuated in the 1X1S, XST, SLI and EDI groups. As shown in Figure 2D, there was a marked decline in I/R neurological score, while the 1X1S, XST, SLI and EDI treatments resulted in a statistically significant improvement in neurobehavioral deficits compared with the MOD group. However, there were no significant changes in the percent survival of the treatment groups compared with the MOD group (Figure 2E). When compared with the EDI group, the SLI, XST and 1X1S groups exhibited no significantly changed body weight and neuro- logical scores. The $1 / 2 \times 1 / 2 S$ treatment group exhibited no significantly increased body weight and decreased neurobehavioral deficits compared with the MOD group.

\section{X1S increases regional cerebral blood flow ( $\mathrm{rCBF}$ ) in I/R-treated} rats

The rCBF was determined by a laser Doppler flowmetry system in the different groups, as shown in Figure 3A-3G, and the quantification of the results is shown in Figure $3 \mathrm{H}$. The administration of EDI, SLI, XST and 1X1S resulted in a significant increase in $\mathrm{rCBF}$ at $72 \mathrm{~h}$ after reperfusion versus the MOD group. There was a significant decrease in the MOD group versus the NOR group. When compared to the EDI group, the SLI, XST and 1X1S groups exhibited no significant increases in rCBF. The $1 / 2 X 1 / 2 S$ treatment group showed no significant increases in $\mathrm{rCBF}$ compared with the MOD group.

1X1S reduces lesion volumes and ameliorates histopathological damage in I/R-treated rats

Lesion volumes were evaluated in mouse brains by crystal violet staining after I/R injury. Representative samples of crystal violet-stained brain sections are shown in Figure 4A, with cor-

\section{A}

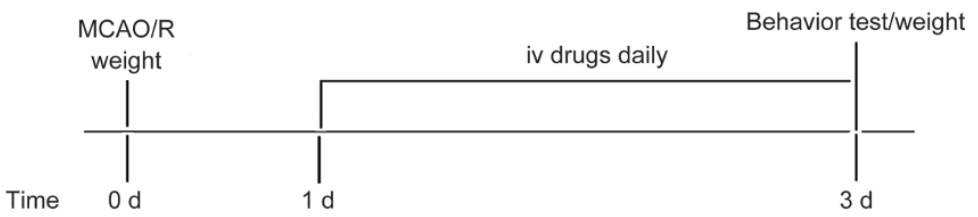

B
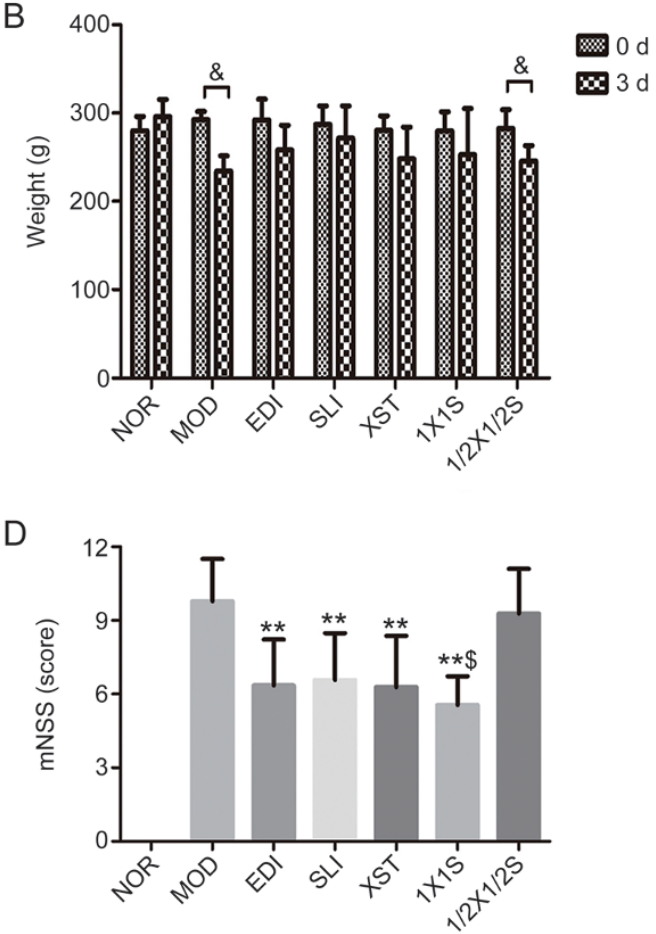

C

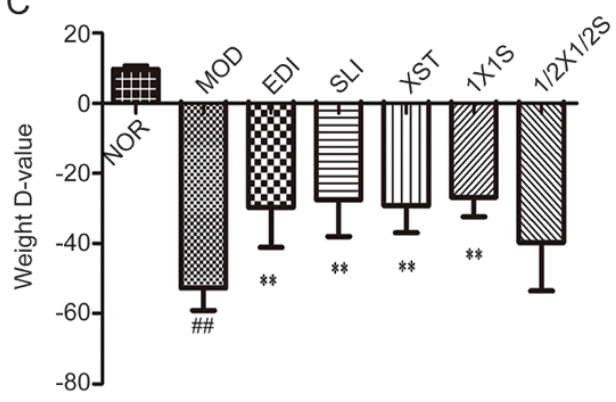

E

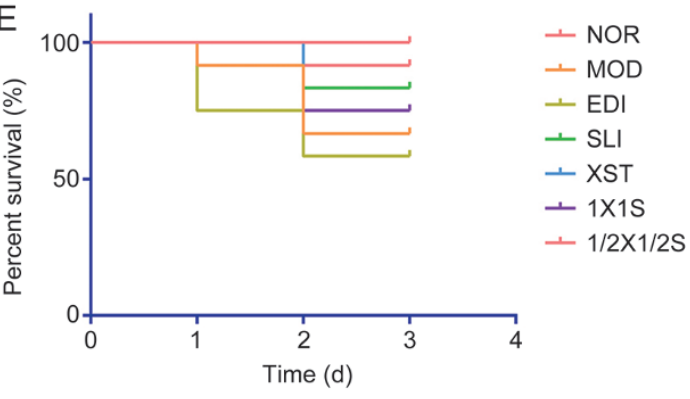

Figure 2. Effects of $1 X 1 S$ on brain injury in rats with cerebral I/R. (A) Schematic of the experiment protocol, (B, C) body weight changes, (D) neurobehavioral outcomes, (E) percent survival. Rats were subjected to $1.5 \mathrm{~h}$ of ischemia and $72 \mathrm{~h}$ of reperfusion. EDI, SLI, XST, 1X1S and 1/2X $1 / 2 \mathrm{~S}$ was administered $3 \mathrm{~d}$ after reperfusion, $n=41$. ${ }^{\# \#} P<0.01$ vs NOR. ${ }^{* *} P<0.01$ vs MOD. ${ }^{\circledR} P<0.05$ vs 0 d. ${ }^{\$} P<0.05$ vs SLI and XST. 

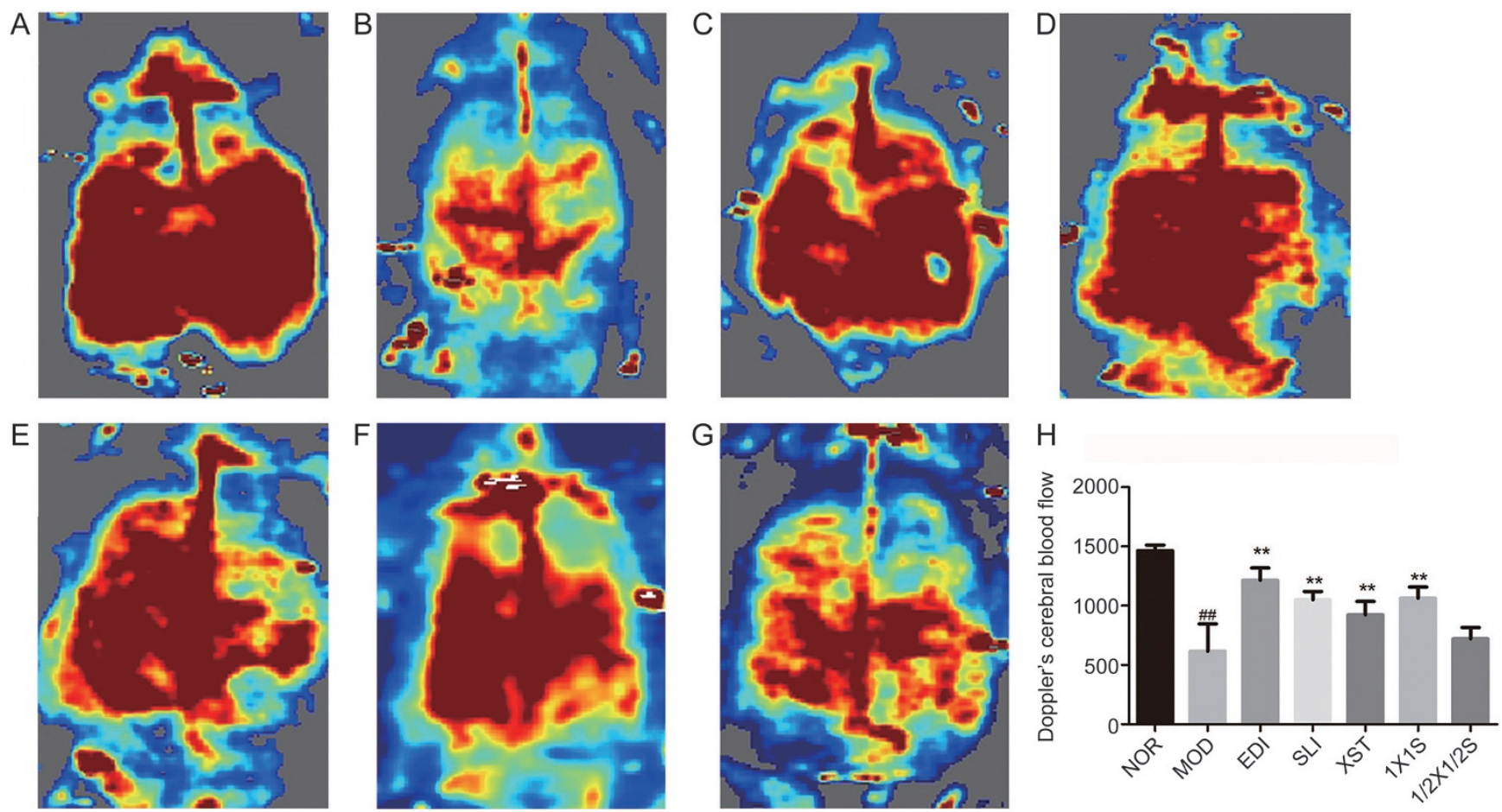

Figure 3. Effect of $1 X 1 S$ on regional cerebral blood flow in rats with cerebral I/R. (A-G) The representative images of cerebral blood flow of ipsilateral cortex. A-G were NOR, MOD, EDI, SLI, XST, $1 \mathrm{X} 1 \mathrm{~S}$ and $1 / 2 \mathrm{X} 1 / 2 \mathrm{~S}$ groups, respectively. The magnitude of rCBF is represented by different colors, with blue to red denoting low to high. (H) Quantitative analysis of rCBF in different groups. $n=16$. ${ }^{\# \#} P<0.01$ vs NOR. ${ }^{* *} P<0.01$ vs MOD.

responding lesion volumes and statistical data being shown in Figure 4B. Lesion volumes were significantly increased in the I/R operative group, whereas SLI, XST, EDI and 1X1S reduced the lesion volumes induced by I/R. Figure $4 \mathrm{C}$ shows the histopathological changes after $1.5 \mathrm{~h}$ of occlusion and $72 \mathrm{~h}$ of reperfusion by H\&E staining, and the result showed that SLI, XST, EDI and 1X1S treatment ameliorated histopathological damage in I/R-treated rats. Compared with the EDI group, the SLI, XST and 1X1S groups exhibited no significant reductions in lesion volumes. The 1X1S treatment group showed significant decreases in lesion volumes compared with the SLI group and the XST group. Moreover, the lesion volumes in the $1 / 2 X 1 / 2 S$ group showed no decrease compared with the MOD group.

\section{X1S attenuated neuronal injury after I/R-treated rats}

Nissl staining was used to reveal neuronal injury in the penumbra of ischemic cortex at $3 \mathrm{~d}$ after treatment. In the NOR group, normal neurons had normal morphology with a clear nucleolus, abundant cytoplasm, and intact structure, which were arranged orderly. In the MOD group, most of neurons in the ischemic penumbra had enlarged intercellular spaces were disappearance of the Nissl body, were shrunken and deep stained, and/or had nuclear pyknosis, which are the indications of the injury induced by I/R. However, these characteristic morphological changes were not observed in the NOR group. Compared to the SLI group and the XST group, these characteristic morphological changes were rarely observed in the 1X1S group due to the neuroprotective effect of SLI com- bined with XST (Figure 5). Compared with the EDI group, the SLI, XST and 1X1S groups exhibited no significant increases in the number of intact cells. Moreover, the number of intact cells in the $1 / 2 X 1 / 2 S$ group had not been increased compared with the MOD group.

1X1S elevates the levels of SOD, CAT and GSH and reduces the level of MDA in I/R-treated rats

Antioxidant enzyme (SOD, CAT and GSH) levels and MDA content were examined in order to assess the protective effect of 1X1S treatment on I/R rats. As shown in Figure 6, after I/R injury, higher level of MDA and lower levels of SOD, CAT and GSH were present in the MOD group compared with NOR group. SLI, XST, EDI and 1X1S treatment significantly elevated the levels of SOD, CAT and GSH and reduced the level of MDA in brain tissue compared with the MOD group. Treatment of 1X1S could upregulate the levels of SOD, CAT and GSH and downregulate the level of MDA compared with the SLI group and the XST group. When compared with the EDI group, higher level of MDA and lower levels of SOD, CAT and GSH were present in the SLI group and the XST group.

\section{X1S reduces the level of reactive oxygen species (ROS) in} I/R-treated rats

Previous studies have demonstrated that ROS plays a key role in the pathophysiological response of the brain after $\mathrm{I} / \mathrm{R}$ injury $^{[32]}$. The results showed that I/R injury significantly increased ROS production compared to the normal rats. Conversely, the I/R rats that received SLI, XST, EDI and 1X1S 

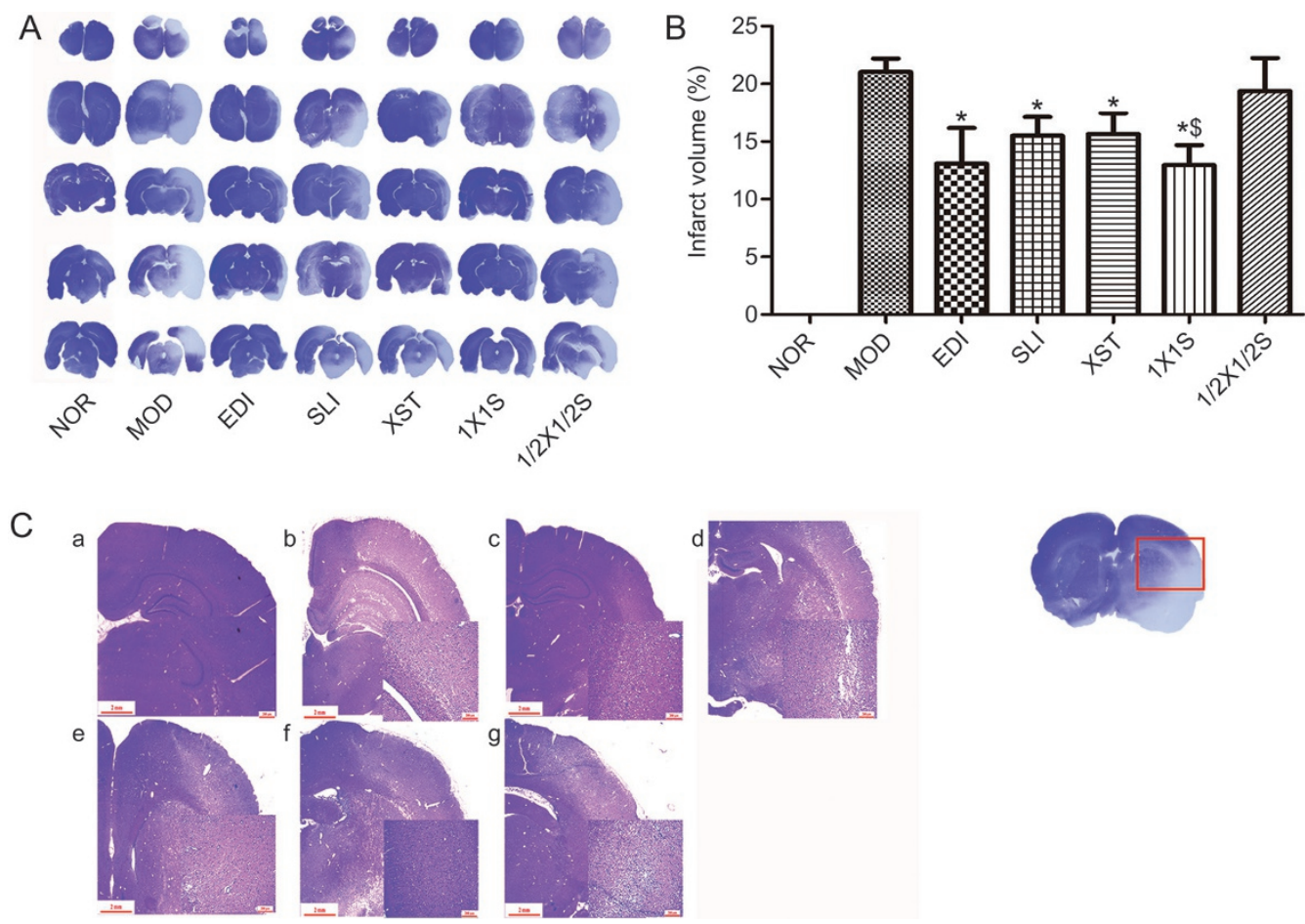

Figure 4. Effect of $1 \mathrm{X} 1 \mathrm{~S}$ on lesion volume and histopathological changes of brain sections in rats with cerebral I/R. (A, B) Infarct volumes, (C) Hematoxylin-and-eosin-stained slides of the brain sections of rat in different groups were examined under a light microscope. a-g were NOR, MOD, EDI, SLI, XST, 1 X1S and $1 / 2 X 1 / 2 S$ groups, respectively. ${ }^{*} P<0.05$ vs MOD. ${ }^{\$} P<0.05$ vs SLI and XST. Scale bar=100 $\mu \mathrm{m} . n=12$.

treatment had significantly less ROS generation level in comparison to the I/R rats. Compared with the EDI group, higher levels of ROS were present in the SLI group and the XST group. Treatment of 1X1S could decrease the level ROS compared with the SLI group and the XST group (Figure 7).

1X1S suppressed activation of microglia and astrocytes in the I/R-treated rats

Neuronal death in cerebral I/R injury is associated with astrocytosis and microgliosis. The counted number of microglia and astrocyte cells that positively reacted to IBA-1 and GFAP were markedly increased in the ipsilateral hippocampus of $\mathrm{MCAO} / \mathrm{R}$ rats (Figure 8A, 8B). Compared to that in MOD rats, decreases of IBA-1 (Figure 8B) and GFAP (Figure 8II) immunoreactivity were found in the ipsilateral hippocampus of SLI-, XST- and 1X1S-treated MCAO/R rats. Figure 8C-8E shows that stroke upregulated brain level of GFAP and IBA-1 protein and mRNA, and SLI, XST and 1X1S treatment reduced their expression in MOD rats. The MOD rats had significantly higher levels of GFAP and IBA-1 protein and mRNA than
A

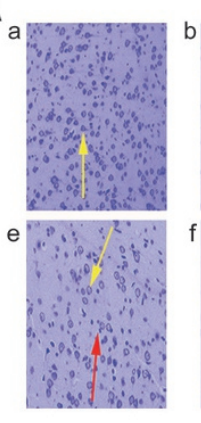

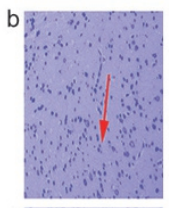

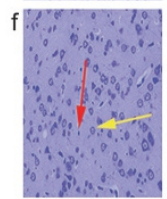

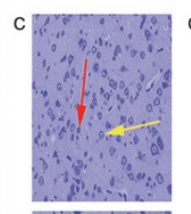

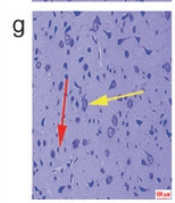

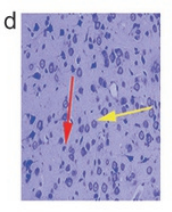

B

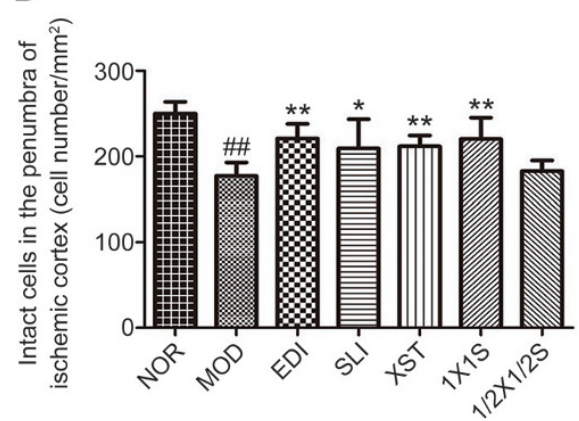

Figure 5. Representative pictures of Nissl staining at $3 \mathrm{~d}$ after treatment and relevant quantitative analysis. (A) The pictures of Nissl staining (200× magnifications). a-g were NOR, MOD, EDI, SLI, XST, 1X1S and 1/2X1/2S groups, respectively. Normal neurons had normal morphology with clear nucleolus, abundant cytoplasm, and intact structure (yellow arrow). Abnormal neurons appeared shrunken and deep stained (red arrow). (B) Quantitative analysis of intact cells in penumbra of ischemic area at $3 \mathrm{~d}$ after treatment. Data are presented as the mean \pm SD. $n=12$. ${ }^{\# \#} P<0.01 \mathrm{vs}$ NOR, ${ }^{*} P<0.05,{ }^{* *} P<0.01$ vs MOD. 

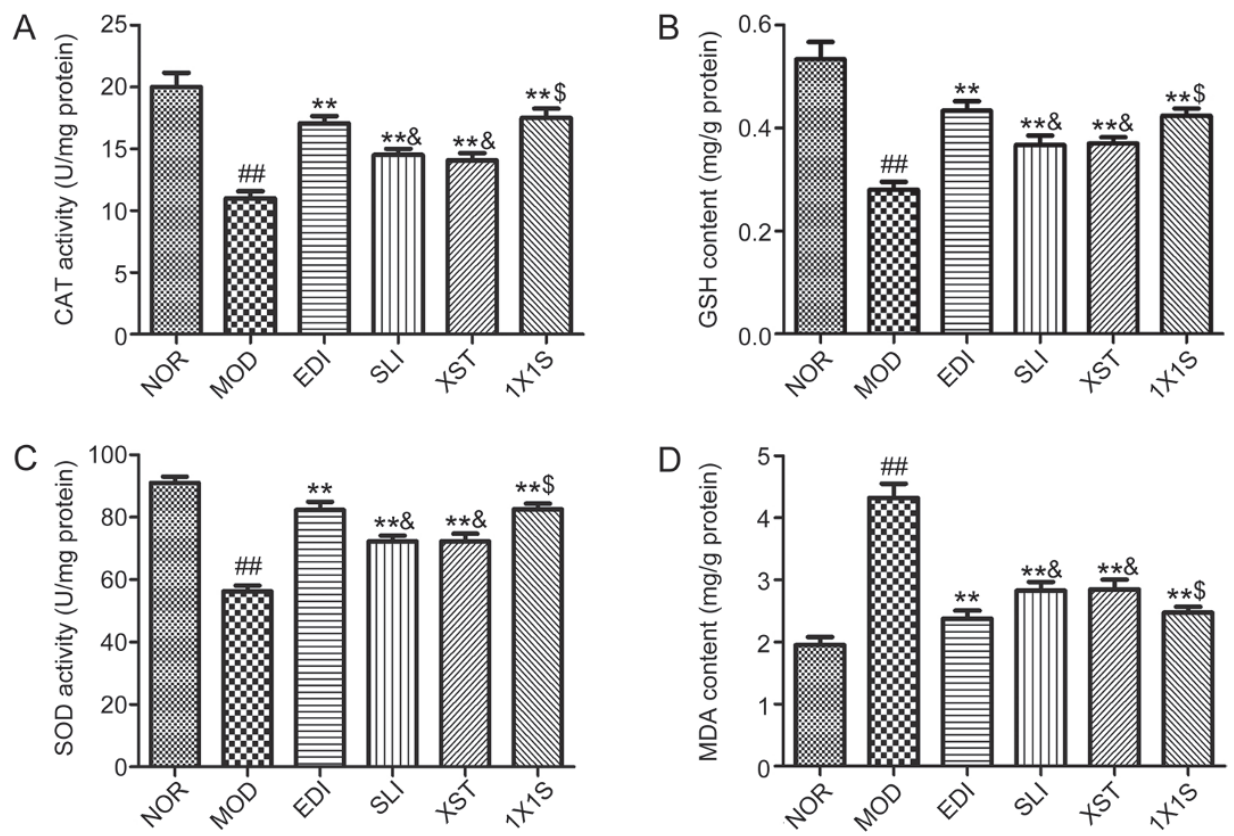

Figure 6. Effect of $1 \mathrm{X} 1 \mathrm{~S}$ treatment on catalase (CAT; A), glutathione-S-transferase (GSH; B), superoxide dismutase (SOD; C) and malondialdehyde (MDA; D) in rats with cerebral I/R. Data are represented as mean \pm SD. $n=5 .{ }^{\# \#} P<0.01$ vs NOR. ${ }^{* *} P<0.01$ vs MOD. ${ }^{\$} P<0.05$ vs SLI and XST. ${ }^{\&} P<0.05$ vs EDI.

NOR animals and SLI-, XST- and 1X1S-treated animals. We found that the 1X1S group had no significant difference compared with SLI and XST in levels of GFAP and IBA-1 proteins but had a significant difference in levels of GFAP and IBA-1 mRNA. These results suggest that SLI, XST and 1X1S could inhibit the activation of both microglia and astrocytes induced by cerebral ischemia/reperfusion.

1X1S treatment significantly increased the levels of HO-1, NQO-1 and Nrf-2 and reduced Keap1 expression in I/R-treated rats

To understand the mechanism of $1 \mathrm{X} 1 \mathrm{~S}$ on cerebral protection, the expression of Nrf-2, HO-1, NQO-1 and Keap1 in I/ R rat brain tissues was measured using Western blotting and realtime qPCR (Figure 9A-9C). The Western blotting and realtime qPCR results showed that the SLI, XST, EDI and 1X1S treatments significantly increased the expression of HO-1,

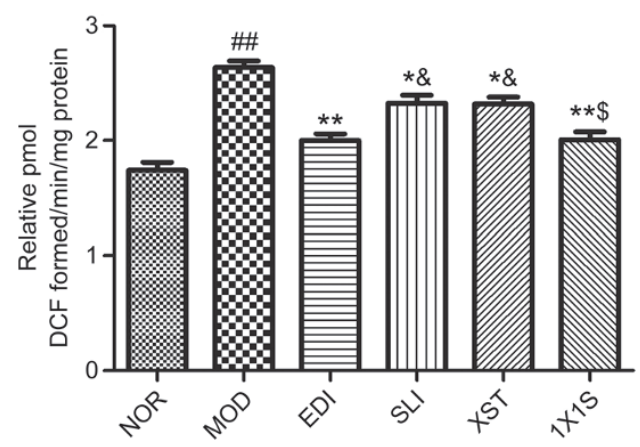

Figure 7. Effect of $1 X 1 S$ treatment on ROS in rats with cerebral I/R. Data are represented as mean \pm SD. $n=5$. ${ }^{\#} P<0.01$ vs NOR. ${ }^{*} P<0.05$ and ${ }^{* *} P<0.01$ vs MOD. ${ }^{\$} P<0.05$ vs SLI and XST. ${ }^{\circledR} P<0.05$ vs EDI.
NQO-1 and Nrf-2 and reduced the expression of the Keap1 in the ischemic brains compared with the MOD group. 1X1S remarkably upregulated the expression of Nrf-2, HO-1 and NQO-1 compared with the XST and downregulated the expression of Keap1 compared with the SLI and XST.

\section{X1S altered Nrf-2 and Keap1 expression in I/R-treated rats}

To clarify whether the Nrf-2/Keap1 pathway was involved in the antioxidative stress response of 1X1S, we examined the cytoplasmic expression and nuclear translocation of Keap1 and Nrf-2 in I/R-treated rat brain tissues (Figure 10). According to the immunofluorescence analysis, in the NOR group, Nrf-2 was localized to the cytoplasm. In SLI-, XST-, EDI- and 1X1S-treated groups, downregulated Nrf-2 expression with cytoplasmic localization and a subsequent profound upregulated Nrf-2 expression was observed in the nucleus. In SLI-, XST-, EDI- and 1X1S-treated rats, a nuclear translocation of Nrf-2 and a decrease in Keap1 expression were observed. However, 1X1S remarkably downregulated the expression of Keap1 and facilitated the nuclear translocation of Nrf-2 compared with XST. These results indicate that SLI is major factor in the antioxidative effects of 1X1S.

\section{Discussion}

In the present study, we used an $\mathrm{MCAO} / \mathrm{R}$ rat model to determine the effects of 1X1S following acute ischemic stroke through the activation of the Nrf-2/Keap1 antioxidant pathway. Our results show that body weight, neurobehavioral deficits, lesion size, glial activation, $\mathrm{rCBF}$ and neuronal injury were decreased in this model by SLI, XST, EDI and 1X1S treatment. Additionally, the treated rats exhibited decreased ROS and MDA activities and increased levels of SOD, CAT 

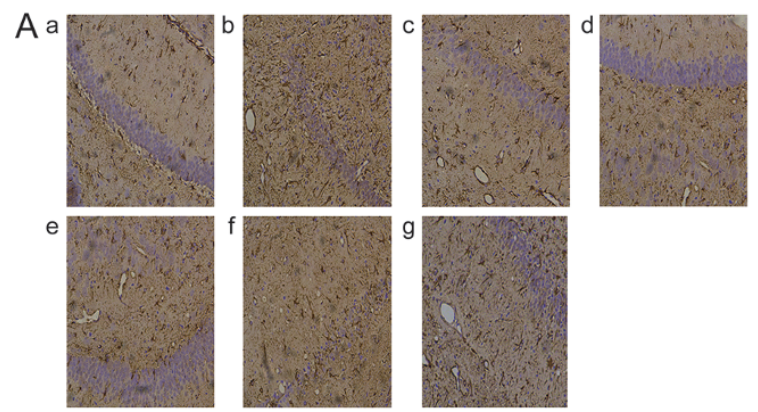

C

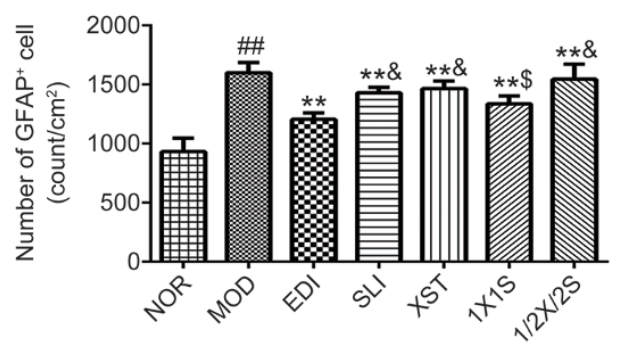

E

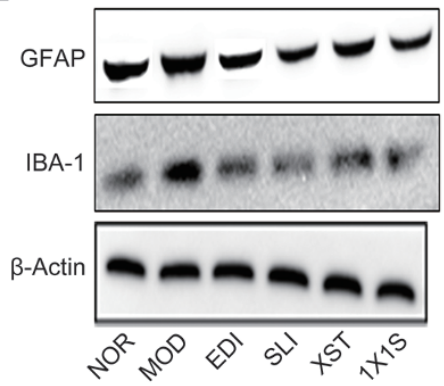

F
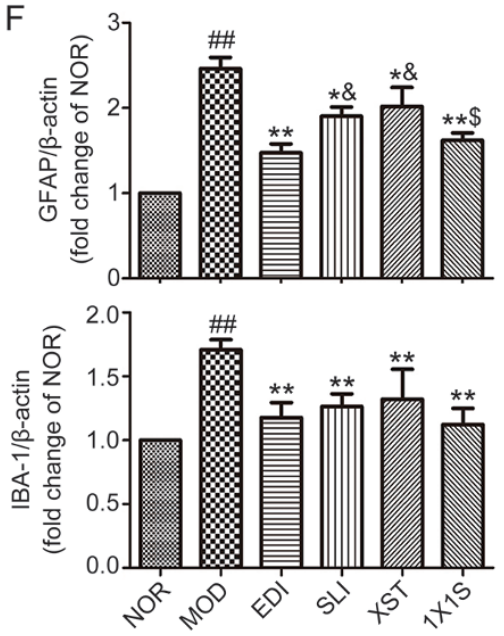
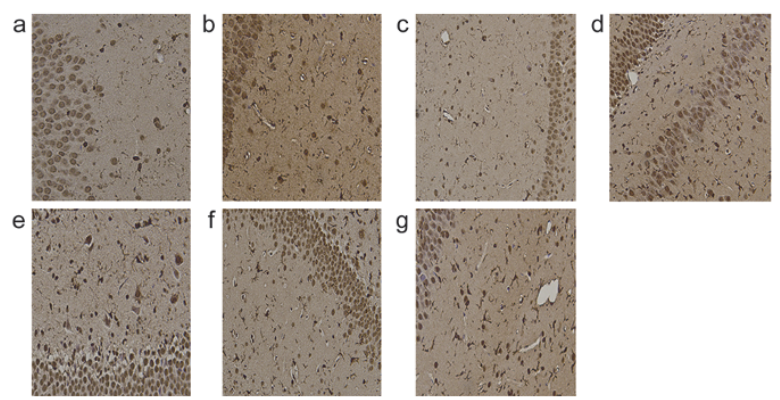

D

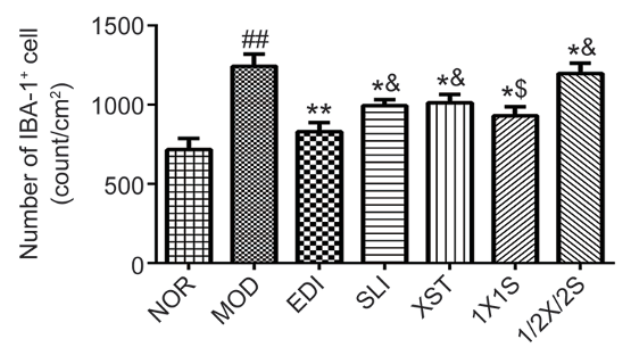

Figure 8. $1 \mathrm{X} 1 \mathrm{~S}$ inhibited the activation of microglia and astrocyte in the hippocampus area of middle cerebral artery occlusion/reperfusion (MCAO/R) rats. (A, B) Immunohistochemistry for GFAP and IBA-1in the hippocampus. Scale bar: $100 \mu \mathrm{m}$. a-g were NOR, MOD, EDI, SLI, XST, 1X1S and 1/2X1/2S groups, respectively. (C, D) Statistical analysis of total number of IBA-1 and GFAP positive cells in the hippocampus. (E) GFAP and IBA-1 proteins expression in MCAO/R rats. (F) Bar graph showed a quantification of GFAP and IBA-1 expression. (G) GFAP and IBA-1 mRNA expression in MCAO/R rats. Data are presented as mean \pm SD from 4 experiments. ${ }^{\#} P<0.05$, ${ }^{\# \#} P<0.01$ vs NOR. ${ }^{*} P<0.05,{ }^{* *} P<0.01$ vs MOD. ${ }^{\$} P<0.05$ vs SLI and XST. ${ }^{\&} P<0.05$ vs EDI.

and GSH compared to the MOD group. Furthermore, SLI combined with XST protected the brain more effectively than the SLI and XST used alone. Furthermore, 1X1S and edaravone post-treatment remarkably reduced infarct volume and improved neurological function. These results indicated that 1X1S could be a potential clinical cerebral I/R injury therapy.

SLI, a water-soluble component of Salviae miltiorrhizae roots (Danshen in Chinese) and has been widely used in China for the treatment of cerebrovascular conditions, such as ischemic stroke $^{[33]}$. XST, a freeze-dried saponin powder injection of Panax notoginseng Saponin, is used to treatment stroke, chest stuffiness and pains, and central retinal vein occlusion ${ }^{[34]}$. Many studies suggest that SLI and XST have strong scavenging activity against oxidative stress ${ }^{[18,20,33]}$. Although those reported that the SLI and XST used alone have oxidative stress effect, there is no report on the effect of SLI and XST protecting against ischemic injury in $\mathrm{MCAO} / \mathrm{R}$ rats through its suppression of oxidative stress. However, oxidative stress has been found to play a crucial role in ischemic stroke ${ }^{[35,36]}$. EDI, a potent scavenger of hydroxyl radicals, is widely used to treat acute ischemic stroke ${ }^{[37]}$. Therefore, in the current study, the antioxidative effects of SLI, XST and EDI in cerebral I/R injuries were compared, and possible mechanisms of the brainprotective effect of SLI and XST were suggested. Additionally, we wanted to investigate whether the combined use strategy can compensate for their own disadvantages for the treatment of stroke or have stronger effects than the drugs used alone and designed $1 \mathrm{X} 1 \mathrm{~S}$ and 1/2X1/2S groups. 
B
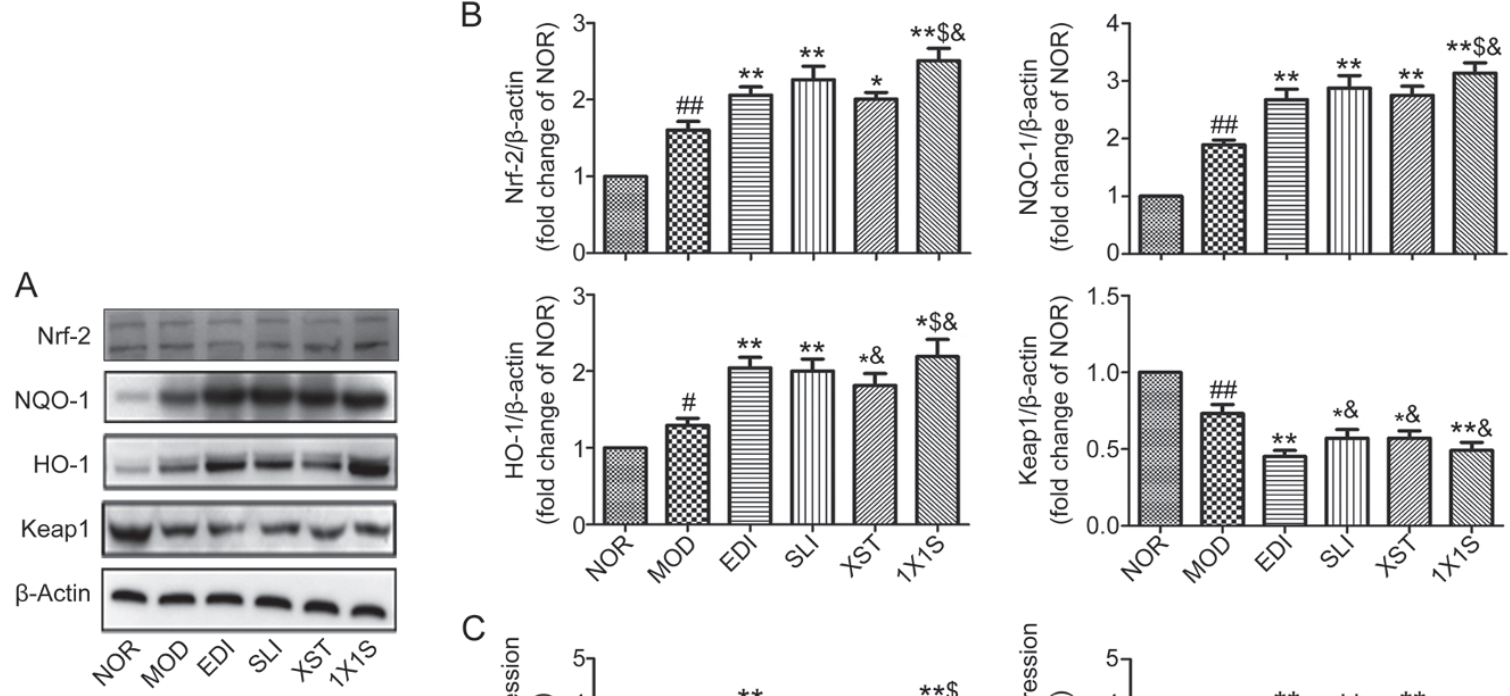

C
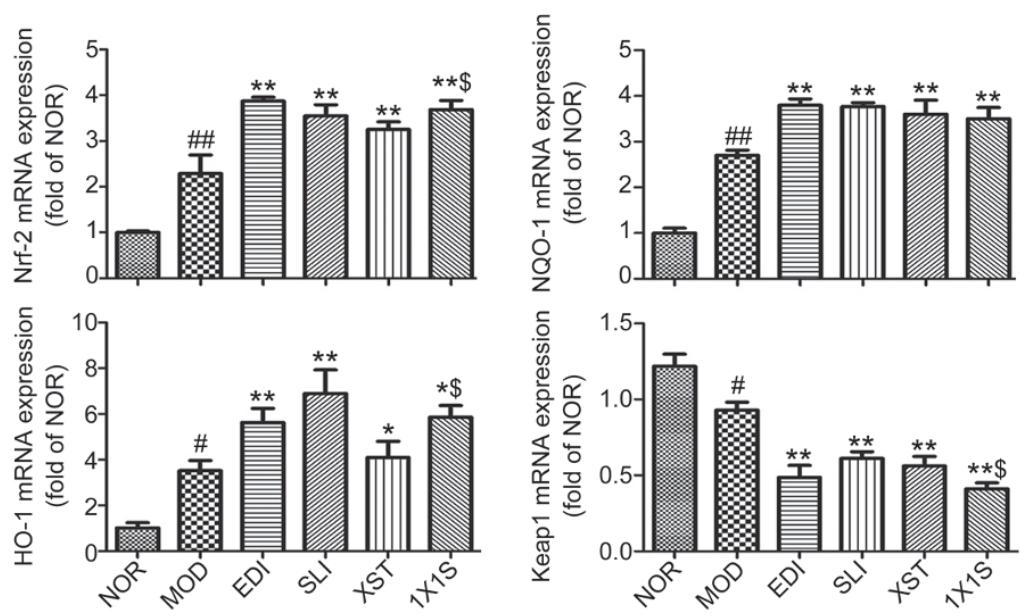

Figure 9. 1X1S increases in the expression level of Nrf-2, HO-1, NQO-1 and decreases in the level of Keap1 proteins and mRNA after I/R treated rats when compared to the MOD group. (A) Expressions of Nrf-2, HO-1, NQO-1 and Keap1 proteins were measured with Western blot. (B) Densitometric analysis was performed using the Quantity One software. (C) Expressions of Nrf-2, HO-1, NQO-1 and Keap1 mRNA were measured with PCR. Data are presented as mean \pm SD from 3 experiments. ${ }^{\#} P<0.05,{ }^{\# \#} P<0.01$ vs NOR. ${ }^{*} P<0.05,{ }^{* *} P<0.01$ vs MOD. ${ }^{\$} P<0.05$ vs XST. ${ }^{\circledR} P<0.05$ vs SLI and XST.

Our results show that SLI and XST did not have significant differences compared with EDI, while 1X1S protected the brain more effectively than the SLI and XST used alone in $\mathrm{MCAO} / \mathrm{R}$ rats. However, $1 / 2 \mathrm{X} 1 / 2 \mathrm{~S}$ did not improve body weight, neurobehavioral deficits, lesion size, glial activation, $\mathrm{rCBF}$ and neuronal injury in the MCAO/R rats compared with the MOD group.

Reactive gliosis associated with ischemic stroke involves both astrocytes and microglia, which is an important component of the cellular and molecular pathways involved in stroke-induced destructive responses ${ }^{[38]}$. In our study, $\mathrm{MCAO} / \mathrm{R}$ injury increased the expression of GFAP and IBA-1 expression, while the expression of these markers was reduced in rats treated with SLI, XST, EDI and 1X1S. These observations suggest that the neuroprotective properties of SLI, XST, EDI and IXIS were caused by inhibiting the proliferation of glial cells.

The process of ischemia-reperfusion is multifactorial, and there are several mechanisms involved in the pathogenesis. There is ample evidence that I/R injury is in part caused by an excessive generation of $\operatorname{ROS}^{[7]}$. I/R produces excess ROS while downregulating the expression of SOD, CAT and GSH, resulting in oxidative stress in the brain. Oxidative stress plays a central role among the various factors contributing to $\mathrm{I} / \mathrm{R}$ brain damage ${ }^{[39]}$. Nrf-2 is a key regulator of endogenous antioxidant defense ${ }^{[40]}$. HO-1 is a rate-limiting enzyme ${ }^{[41]}$. At the transcriptional level, HO-1 and other antioxidant enzymes, such as NQO-1, SOD, CAT, GSH and MDA, are mediated by the transcription factor $\mathrm{Nrf-}-2^{[42,43]}$. Under physiological situations, Nrf-2 is bound by Keap1 and resides in the cytoplasm before it is targeted for proteasomal degradation ${ }^{[44-46]}$. Recently, several scientific studies have provided experimental evidence that the Nrf-2/Keap1 pathway is a potential therapeutic target in brain injury after ischemic stroke ${ }^{[11,12]}$. Previously, cerebral ischemia/reperfusion injury was confirmed to be related to these genes ${ }^{[47,48]}$. To explore the possible mechanism of 1X1S antioxidant protection, we investigated the effects of 1X1S on the expression of interference of the Nrf-2/ Keap1 pathway genes. Our results showed that 1X1S upregulated expression of HO-1 and NQO-1, significantly elevated 

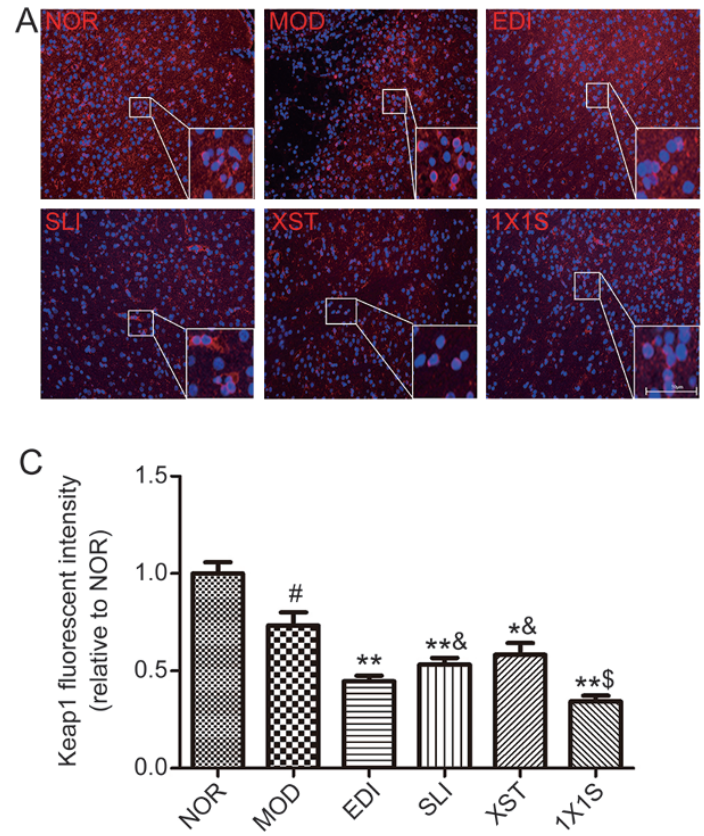

B
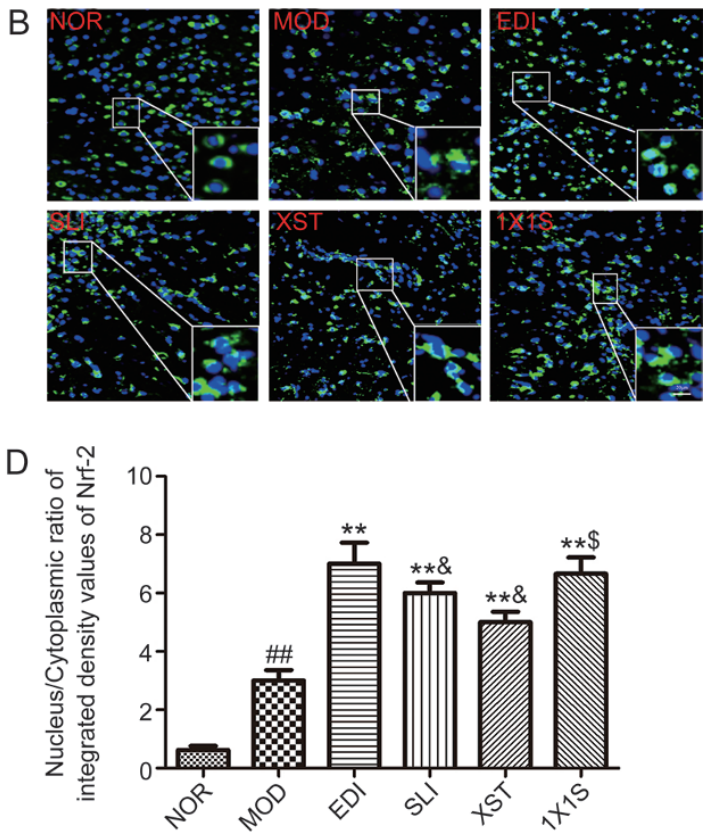

Figure 10. 1X1S significantly decreased the expression of Keap1 and facilitated the nuclear translocation of Nrf-2. (A) Keap1 degradation after 1X1S treatment to I/R rats brain tissue. The analysis was carried out using immunofluorescence staining (magnification, $\times 100$ ); (B) Keap1 dependent Nrf-2 nuclear translocation was observed in I/R rats brain tissue (magnification, $\times 200$ ). (C, D) Bar graph showed a quantification of the expression of Keap1 and nucleus/cytoplasmic ratio of Nrf-2. Data are presented as mean \pm SD. $n=5$. ${ }^{\#} P<0.05,{ }^{\# \#} P<0.01$ vs NOR; ${ }^{*} P<0.05,{ }^{* *} P<0.01$ vs MOD; ${ }^{\circledR} P<0.05$ vs SLI and XST. ${ }^{\$} P<0.05$ vs XST.

the levels of SOD, CAT and GSH, and reduced the levels of MDA and Keap1 in brain tissue when compared with the MOD group. These results suggested that 1X1S could attenuate I/R injury-induced oxidative stress via the Nrf-2/Keap1 pathway.

As previously described ${ }^{[17,28]}$, SLI and XST consist of many bioactive compounds, such as salvianolic acids (B, E), rosmarinic acid and lithospermic acid, and ginsenosides (Rg1, Rb1, $\mathrm{R} 1$, Re and Rd), some of which display neuroprotective effects after cerebral I/R injury. Salvianolic acid B exerts various pharmacological activities, such as anti-inflammation, antioxidation, promotion of cellular proliferation, anti-tumor and preservation of normal cell functions ${ }^{[49]}$. Rosmarinic acid has significant neuroprotective effects during cerebral I/R injury, such as attenuated BBB breakdown, decreased infarct volume and reduced HMGB1 expression in ischemic brain tissue. Ginsenoside $\mathrm{Rb} 1$ weakens the activity of microglia and decreases the upregulation of brain tissue mRNA of TNF- $\alpha$ and interleukin-1 (IL-1), IL- $\beta$, and IL-6 in the brain induced by systemic lipopolysaccharide (LPS) treatment in C57BL/ 6 mice $^{[50,51]}$. Ginsenoside Rg1 attenuates BBB disruption by downregulating the expression of aquaporin 4 induced via ischemic stroke in animals ${ }^{[2]}$. Ginsenoside Rd improves stroke outcome and attenuate mitochondrial dysfunction after transient focal ischemia by reducing inflammatory response and protecting mitochondria ${ }^{[53,54]}$. Notoginsenoside R1 protects the viability of rat neuronal cells in primary cultured mouse cortical neurons induced by glutamate interference ${ }^{[55]}$. Based on these bioactive compounds, it is likely that $1 \mathrm{X} 1 \mathrm{~S}$ could protect against isch- emic stroke through various ways, although our study only explored a part of all pathways.

This current study was conducted to elucidate the mechanism of 1 X1S against focal cerebral I/R injury in rats through attenuation of oxidative stress (Figure 11). I/R injury led to additional ROS production. Excessive ROS caused oxidative stress. Subsequently, under oxidative stress, the Keap1-Nrf-2

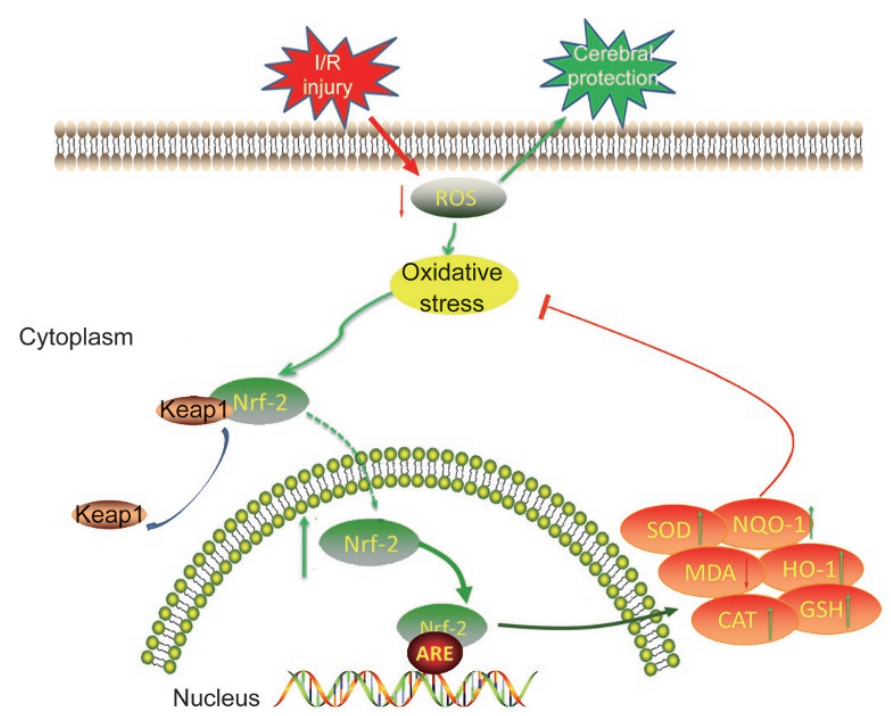

Figure 11. Graphical abstract shows the protective mechanism of 1X1S against cerebral injury following focal cerebral ischemia and reperfusion via Nrf-2/Keap1 pathway. 
complex dissociates and Nrf-2 translocate into the nucleus to bind ARE. Nrf-2 activates antioxidative defense enzymes, including HO-1, NQO-1, SOD, GSH, CAT and MDA, to attenuate oxidative stress. Our findings demonstrated that 1X1S could decrease ROS activities, facilitate the nuclear translocation of Nrf-2 and increase the levels of HO-1, NQO-1, SOD, CAT and GSH and decrease MDA activities. Therefore, 1X1S effectively protected MCAO/R injury against oxidative stress, which was largely dependent on the upregulation of the Nrf-2/Keap1 pathway. It might be the first report that shows that $1 \mathrm{X} 1 \mathrm{~S}$ can potentially ameliorate I/ $\mathrm{R}$ injury-induced oxidative stress in the MCAO/R rat's brain.

Although we demonstrated the cerebral protective effect of 1X1S in MCAO/R rats, we have yet to reveal the underlying mechanisms of $1 \mathrm{X} 1 \mathrm{~S}$ on Nrf-2/Keap1 signaling pathways, which is a limitation of the study. However, it is worthwhile to decipher in future studies.

In conclusion, as illustrated in Figure 11, 1X1S exhibits protective effects against cerebral I/R injury in an MCAO model, which was demonstrated by improved neurological scores, reduced lesion volume and increased regional cerebral blood flow. The mechanism is possibly attributed to activation of the Nrf-2/Keap1 pathway.

\section{Acknowledgements}

This study was supported by the Major New Drugs Innovation and Development (2012zx09101202), the National Natural Sciences Foundation of China (8157140605 and 815740052), the Natural Science Foundation of Tianjin (14JCYBJC28900), the Tianjin Technology Innovation System and the Condition of Platform Construction Plan (16PTSYJC00120).

\section{Author contribution}

Li-min $\mathrm{HU}$ and Fu-jiang WANG conceived and designed the experiments; Fu-jiang WANG and Shao-xia WANG performed the experiments; Fu-jiang WANG, Shao-xia WANG, and Lijuan Chai analyzed the data; Fu-jiang WANG wrote the paper; Fu-jiang WANG, Hong GUO, and Yue ZHANG revised the manuscript. All authors have read and approved the final manuscript.

\section{References}

1 Sarti C, Rastenyte D, Cepaitis Z, Tuomilehto J. International trends in mortality from stroke, 1968 to 1994. Stroke 2000; 31: 1588-601.

2 Roger VL, Go AS, Lloyd-Jones DM, Benjamin EJ, Berry JD, Borden WB, et al. Executive summary: heart disease and stroke statistics--2012 update: a report from the American Heart Association. Circulation 2012; 125: 188-97.

3 Silvestrelli G, Corea F, Paciaroni M, Milia P, Palmerini F, Parnetti L, et al. The Perugia hospital-based Stroke Registry: report of the 2nd year. Clin Exp Hypertens 2002; 24: 485-91.

4 Kwiatkowski TG, Libman RB, Frankel M, Tilley BC, Morgenstern LB, Lu $M$, et al. Effects of tissue plasminogen activator for acute ischemic stroke at one year. N Engl J Med 1999; 340: 1781-7.

5 Aronowski J, Strong R, Grotta JC. Reperfusion injury: demonstration of brain damage produced by reperfusion after transient focal ischemia in rats. J Cereb Blood Flow Metab 1997; 17: 1048-56.
6 Yen TL, Hsu CK, Lu WJ, Hsieh CY, Hsiao G, Chou DS, et al. Neuroprotective effects of xanthohumol, a prenylated flavonoid from hops (Humulus lupulus), in ischemic stroke of rats. J Agric Food Chem 2012; 60: 1937-44.

7 Halladin NL. Oxidative and inflammatory biomarkers of ischemia and reperfusion injuries. Dan Med J 2015; 62: 1-22.

8 Manzanero S, Santro T, Arumugam TV. Neuronal oxidative stress in acute ischemic stroke: sources and contribution to cell injury. Neurochem Int 2013; 62: 712-8.

9 Nguyen T, Nioi P, Pickett CB. The Nrf2-antioxidant response element signaling pathway and its activation by oxidative stress. J Biol Chem 2009; 284: 13291-5.

10 Dinkova-Kostova AT, Talalay P. NAD(P)H:quinone acceptor oxidoreductase 1 (NQO1), a multifunctional antioxidant enzyme and exceptionally versatile cytoprotector. Arch Biochem Biophys 2010; 501: 116-23.

11 Alfieri A, Srivastava S, Siow RC, Cash D, Modo M, Duchen MR, et al. Sulforaphane preconditioning of the Nrf2/HO-1 defense pathway protects the cerebral vasculature against blood-brain barrier disruption and neurological deficits in stroke. Free Radic Biol Med 2013; 65: 1012-22.

12 Shah ZA, Li RC, Ahmad AS, Kensler TW, Yamamoto M, Biswal S, et al. The flavanol (-)-epicatechin prevents stroke damage through the Nrf2/ HO1 pathway. J Cereb Blood Flow Metab 2010; 30: 1951-61.

13 Shang Q, Xu H, Liu Z, Chen K, Liu J. Oral Panax notoginseng preparation for coronary heart disease: a systematic review of randomized controlled trials. Evid Based Complement Alternat Med 2013; 2013: 940125.

14 Lam BY, Lo AC, Sun X, Luo HW, Chung SK, Sucher NJ. Neuroprotective effects of tanshinones in transient focal cerebral ischemia in mice. Phytomedicine 2003; 10: 286-91.

15 Zhuang P, Zhang Y, Cui G, Bian Y, Zhang M, Zhang J, et al. Direct stimulation of adult neural stem/progenitor cells in vitro and neurogenesis in vivo by salvianolic acid B. PLoS One 2012; 7 : e35636.

16 Zhou L, Zuo Z, Chow MS. Danshen: an overview of its chemistry, pharmacology, pharmacokinetics, and clinical use. J Clin Pharmacol 2005; 45: 1345-59.

17 Wang X, Wang S, Wang J, Guo H, Dong Z, Chai L, et al. Neuroprotective effect of Xueshuantong for injection (Lyophilized) in transient and permanent rat cerebral ischemia model. Evid Based Complement Alternat Med 2015; 2015: 134685.

18 Zhao Q, Peng Y, Huang K, Lei Y, Liu HL, Tao YY, et al. Salvianolate protects hepatocytes from oxidative stress by attenuating mitochondrial injury. Evid Based Complement Alternat Med 2016; 2016: 5408705.

19 Fan J, Liu D, He C, Li X, He F. Inhibiting adhesion events by Panax notoginseng saponins and Ginsenoside Rb1 protecting arteries via activation of Nrf2 and suppression of p38-VCAM-1 signal pathway. J Ethnopharmacol 2016; 192: 423-30.

20 Yan JH, Xia X, Zhu YH, Xu F, Zhang L, Zhao Y, et al. Protection effect and mechanism of Compound Xueshuantong Capsule on diabetic nephropathy rats. Zhonghua Yi Xue Za Zhi 2012; 92: 2099-103.

21 Hu B, Duan CH, Yue JH, Ma YL, Zhou DZ, Li DK, et al. Inhibitory effect of total salvianolate lyophilized injection, a herbal preparation, on human cytochrome P450 and P-glycoprotein in vitro and inductive effect on rat CYP1A2 and CYP3A in vivo. Chin J Pharmacol Toxicol 2013; 27: 6-12.

22 Chen X, Guo Y, Hu Y, Yu B, Qi J. Quantitative analysis of highly similar salvianolic acids with ${ }^{[1]} \mathrm{H}$ qNMR for quality control of traditional Chinese medicinal preparation Salvianolate Lyophilized Injection. J 
Pharm Biomed Anal 2016; 124: 281-7.

23 Zhuang P, Wan Y, Geng S, He Y, Feng B, Ye Z, et al. Salvianolic Acids for Injection (SAFI) suppresses inflammatory responses in activated microglia to attenuate brain damage in focal cerebral ischemia. J Ethnopharmacol 2017; 198: 194-204.

24 Fu P, Liu ZY. Determination of the effecient elements of Xuesaitong for injection, Xueshuantong injection and Xuemaitong for injection by fngerprint. West China J Pharm Sci 2007; 22: 76-8.

25 Wang M, Zhang H, Dong R, Tan L, Liu Z, Zhu Y, et al. Compatible stability study of Panax notoginseng saponin injection (Xueshuantong ${ }^{\circledR}$ ) in combination with 47 different injectables. Biomed Chromatogr 2016; 30: 1599-610.

26 Longa EZ, Weinstein PR, Carlson S, Cummins R. Reversible middle cerebral artery occlusion without craniectomy in rats. Stroke 1989; 20: 84-91.

27 Zhao H, Sapolsky RM, Steinberg GK. Interrupting reperfusion as a stroke therapy: ischemic postconditioning reduces infarct size after focal ischemia in rats. J Cereb Blood Flow Metab 2006; 26: 111421.

28 He Q, Wang S, Liu X, Guo H, Yang H, Zhang L, et al. Salvianolate lyophilized injection promotes post-stroke functional recovery via the activation of VEGF and BDNF-TrkB-CREB signaling pathway. Int J Clin Exp Med 2015; 8: 108-22.

29 Chen J, Sanberg PR, Li Y, Wang L, Lu M, Willing AE, et al. Intravenous administration of human umbilical cord blood reduces behavioral deficits after stroke in rats. Stroke 2001; 32: 2682-8.

30 Shah SA, Khan M, Jo MH, Jo MG, Amin FU, Kim MO. Melatonin stimulates the SIRT1/Nrf2 signaling pathway counteracting lipopolysaccharide (LPS)-induced oxidative stress to rescue postnatal rat brain. CNS Neurosci Ther 2017; 23: 33-44.

31 Kuang X, Chen YS, Wang LF, Li YJ, Liu K, Zhang MX, et al. Klotho upregulation contributes to the neuroprotection of ligustilide in an Alzheimer's disease mouse model. Neurobiol Aging 2014; 35: 16978.

32 Huang YY, Huang XQ, Zhao LY, Sun FY, Chen WL, Du JY, et al. CIC3 deficiency protects preadipocytes against apoptosis induced by palmitate in vitro and in type 2 diabetes mice. Apoptosis 2014; 19 : 1559-70.

33 Wang F, He Q, Wang J, Yuan Q, Guo H, Chai L, et al. Neuroprotective effect of salvianolate lyophilized injection against cerebral ischemia in type 1 diabetic rats. BMC Complement Altern Med 2017; 17: 25870.

34 Wang X, Zhu T, Shi L. Analysis on the adverse reaction of Xueshuantong injection. Acta Acad Med Cpaf 2008; 17: 221-22.

35 Wu J, Chen Y, Yu S, Li L, Zhao X, Li Q, et al. Neuroprotective effects of sulfiredoxin-1 during cerebral ischemia/reperfusion oxidative stress injury in rats. Brain Res Bull 2017; 132: 99-108.

36 Zheng Y, Bu J, Yu L, Chen J, Liu H. Nobiletin improves propofolinduced neuroprotection via regulating Akt/mTOR and TLR4/ NF-kappaB signaling in ischemic brain injury in rats. Biomed Pharmacother 2017; 91: 494-503.

37 Yamaguchi T, Awano H, Matsuda H, Tanahashi N. Edaravone with and without $.6 \mathrm{mg} / \mathrm{kg}$ alteplase within 4.5 hours after ischemic stroke: a prospective cohort study (PROTECT4.5). J Stroke Cerebrovasc Dis 2017; 26: 756-65.

38 Yao Y, Chen L, Xiao J, Wang C, Jiang W, Zhang R, et al. Chrysin protects against focal cerebral ischemia/reperfusion injury in mice through attenuation of oxidative stress and inflammation. Int J Mol Sci 2014; 15: 20913-26.

39 Suda S, Katsura K, Kanamaru T, Saito M, Katayama Y. Valproic acid attenuates ischemia-reperfusion injury in the rat brain through inhibition of oxidative stress and inflammation. Eur J Pharmacol 2013; 707: 26-31.

40 Ding $Y$, Chen M, Wang M, Wang M, Zhang T, Park J, et al. Neuroprotection by acetyl-11-keto-beta-Boswellic acid, in ischemic brain injury involves the Nrf2/HO-1 defense pathway. Sci Rep 2014; 4: 1-9.

41 Siow RC, Sato H, Mann GE. Heme oxygenase-carbon monoxide signalling pathway in atherosclerosis: anti-atherogenic actions of bilirubin and carbon monoxide? Cardiovasc Res 1999; 41: 385-94.

42 Siow RC, Ishii T, Mann GE. Modulation of antioxidant gene expression by 4-hydroxynonenal: atheroprotective role of the Nrf2/ARE transcription pathway. Redox Rep 2007; 12: 11-5.

43 Wei Y, Gong J, Yoshida T, Eberhart CG, Xu Z, Kombairaju P, et al. Nrf2 has a protective role against neuronal and capillary degeneration in retinal ischemia-reperfusion injury. Free Radic Biol Med 2011; 51: 216-24.

44 Stepkowski TM, Kruszewski MK. Molecular cross-talk between the NRF2/KEAP1 signaling pathway, autophagy, and apoptosis. Free Radic Biol Med 2011; 50: 1186-95.

45 Cheng X, Siow RC, Mann GE. Impaired redox signaling and antioxidant gene expression in endothelial cells in diabetes: a role for mitochondria and the nuclear factor-E2-related factor 2-Kelch-like ECH-associated protein 1 defense pathway. Antioxid Redox Signal 2011; 14: 469-87.

46 Jeong WS, Jun M, Kong AN. Nrf2: a potential molecular target for cancer chemoprevention by natural compounds. Antioxid Redox Signal 2006; 8: 99-106.

47 Yu SS, Zhao J, Lei SP, Lin XM, Wang LL, Zhao Y. 4-Hydroxybenzyl alcohol ameliorates cerebral injury in rats by antioxidant action. Neurochem Res 2011; 36: 339-46.

48 Shah ZA, Nada SE, Dore S. Heme oxygenase 1, beneficial role in permanent ischemic stroke and in Gingko biloba (EGb 761) neuroprotection. Neuroscience 2011; 180: 248-55.

49 Wang SX, Hu LM, Gao XM, Guo H, Fan GW. Anti-inflammatory activity of salvianolic acid $B$ in microglia contributes to its neuroprotective effect. Neurochem Res 2010; 35: 1029-37.

50 Chen W, Guo Y, Yang W, Zheng P, Zeng J, Tong W. Protective effect of ginsenoside Rb1 on integrity of blood-brain barrier following cerebral ischemia. Exp Brain Res 2015; 233: 2823-31.

51 Lee JS, Song JH, Sohn NW, Shin JW. Inhibitory effects of ginsenoside $\mathrm{Rb} 1$ on neuroinflammation following systemic lipopolysaccharide treatment in mice. Phytother Res 2013; 27: 1270-6.

52 Zhou Y, Li HQ, Lu L, Fu DL, Liu AJ, Li JH, et al. Ginsenoside Rg1 provides neuroprotection against blood brain barrier disruption and neurological injury in a rat model of cerebral ischemia/reperfusion through downregulation of aquaporin 4 expression. Phytomedicine 2014; 21: 998-1003.

53 Ye R, Kong X, Yang Q, Zhang Y, Han J, Zhao G. Ginsenoside Rd attenuates redox imbalance and improves stroke outcome after focal cerebral ischemia in aged mice. Neuropharmacology 2011; 61: 81524.

54 Ye R, Zhang X, Kong X, Han J, Yang Q, Zhang Y, et al. Ginsenoside Rd attenuates mitochondrial dysfunction and sequential apoptosis after transient focal ischemia. Neuroscience 2011; 178: 169-80.

55 Gu B, Nakamichi N, Zhang WS, Nakamura Y, Kambe Y, Fukumori R, et al. Possible protection by notoginsenoside R1 against glutamate neurotoxicity mediated by $N$-methyl- $D$-aspartate receptors composed of an NR1/NR2B subunit assembly. J Neurosci Res 2009; 87: 214556. 